\title{
AK 3012
}

National Cancer Institute

\section{Source}

National Cancer Institute. AK 3012. NCI Thesaurus. Code C104266.

A proprietary topical formulation. Upon subcutaneous administration, the active ingredient in AK 3012 may inhibit actinic keratosis. 